\title{
On radial behaviour and balanced Bloch functions
}

\section{Juan Jesús Donaire and Christian Pommerenke}

Abstract. A Bloch function $g$ is a function analytic in the unit disk such that $\left(1-|z|^{2}\right)\left|g^{\prime}(z)\right|$ is bounded. First we generalize the theorem of Rohde that, for every "bad" Bloch function, $g(r \zeta)(r \longrightarrow 1)$ follows any prescribed curve at a bounded distance for $\zeta$ in a set of Hausdorff dimension almost one. Then we introduce balanced Bloch functions. They are characterized by the fact that $\left|g^{\prime}(z)\right|$ does not vary much on each circle $\{|z|=r\}$ except for small exceptional arcs. We show e.g. that

$$
\int_{0}^{1}\left|g^{\prime}(r \zeta)\right| d r<\infty
$$

holds either for all $\zeta \in \mathbb{T}$ or for none.

\section{Radial behaviour of Bloch functions.}

Let $\mathbb{D}=\{z \in \mathbb{C}:|z|<1\}$ and $\mathbb{T}=\partial \mathbb{D}$. The function $g$ analytic in $\mathbb{D}$ is called a Bloch function if

$$
\|g\|_{\mathcal{B}}=\sup _{z \in \mathbb{D}}\left(1-|z|^{2}\right)\left|g^{\prime}(z)\right|<\infty
$$

This holds if and only if the Riemann image surface of $g$ as a cover of $\mathbb{C}$ does not contain arbitrarily large unramified disks. We denote the family of Bloch functions by $\mathcal{B}$. 
First we generalize a surprising result of Steffen Rohde [Ro93]. Let $c_{1}, c_{2}, \ldots$ be positive absolute constants and let $\operatorname{dim} E$ denote the Hausdorff dimension [Fa85, p. 7] of $E \subset \mathbb{T}$. Note that $\operatorname{dim} \mathbb{T}=1$.

Theorem 1.1. Let $G \subset \mathbb{C}$ be a domain with $0 \in G$ and let $g$ be a Bloch function with $\|g\|_{\mathcal{B}} \leq 1$ and $g(0)=0$. We assume that, for almost all $\zeta \in \mathbb{T}$,

$$
\lim _{r \rightarrow 1} g(r \zeta) \text { lies in } \mathbb{C} \backslash G \text { or does not exist. }
$$

Let $\Gamma$ be any halfopen curve in $G$ starting at 0 . If

$$
c_{1}<R<\operatorname{dist}(0, \partial G), \quad \operatorname{dist}(\Gamma, \partial G) \geq 2 R,
$$

then there exists $E_{\Gamma} \subset \mathbb{T}$ with

$$
\operatorname{dim} E_{\Gamma} \geq 1-\frac{c_{2}}{R}
$$

such that, for $\zeta \in E_{\Gamma}$, we can find a parametrization $\gamma_{\zeta}(r), 0 \leq r<1$ of $\Gamma$ with $\gamma_{\zeta}(0)=0$ such that

$$
\left|g(r \zeta)-\gamma_{\zeta}(r)\right| \leq 2 R, \quad \text { for } 0 \leq r<1 .
$$

This theorem is due to Rohde [Ro93] for the case that $G=\mathbb{C}$. Thus the radial image follows any prescribed curve with a bounded deviation on a set of dimension almost 1 . Now we apply this theorem to (injective) conformal maps $f$ of $\mathbb{D}$ into $\mathbb{C}$. It is well-known [DuShSh66], [Be72] that

$$
\begin{aligned}
& f \text { conformal implies }\left\|\log f^{\prime}\right\|_{\mathcal{B}} \leq 6 \\
& \left\|\log f^{\prime}\right\|_{\mathcal{B}} \leq 1 \text { implies } f \text { conformal }
\end{aligned}
$$

If the radial limit $f(\zeta)$ exists and is finite (which holds for almost all $\zeta \in \mathbb{T})$, we write

$$
\begin{aligned}
& \alpha(\zeta)=\liminf _{r \rightarrow 1} \arg ((r \zeta)-f(\zeta)), \\
& \beta(\zeta)=\limsup _{r \rightarrow 1} \arg (f(r \zeta)-f(\zeta)) .
\end{aligned}
$$

We give a partial generalization of [CaPo97, Theorem 1]. 
Corollary 1.2. Let $f$ map $\mathbb{D}$ conformally into $\mathbb{C}$ and suppose that

$$
\begin{aligned}
& \limsup _{r \rightarrow 1}\left|f^{\prime}(r \zeta)\right| \geq 1, \quad \text { for almost all } \zeta \in \mathbb{T}, \\
& \liminf _{r \rightarrow 1}\left|f^{\prime}\left(r \zeta_{0}\right)\right|=0, \quad \text { for some } \zeta_{0} \in \mathbb{T} .
\end{aligned}
$$

Then, for $j=1,2,3,4$, there exist sets $E_{j} \subset \mathbb{T}$ with $\operatorname{dim} E_{j}=1$, such that

i) $\alpha(\zeta)=-\infty, \beta(\zeta)=+\infty$, for $\zeta \in E_{1}$ (twist point),

ii) $\alpha(\zeta)=\beta(\zeta)=+\infty$, for $\zeta \in E_{2}$ (spiral point),

iii) $-\infty<\alpha(\zeta)<\beta(\zeta)=+\infty$, for $\zeta \in E_{3}$ (gyration point),

iv) $-\infty<\alpha(\zeta)+2 \pi<\beta(\zeta)<+\infty$, for $\zeta \in E_{4}$ (oscillation point).

Moreover $f(\zeta)$ is well-accessible for $\zeta \in E_{j}(j=1,2,3,4)$.

The McMillan Twist Theorem [Mc69], [Po92, p. 142] states that, for almost all points $\zeta \in \mathbb{T}$, either $\zeta$ is a twist point or the angular derivative $f^{\prime}(\zeta) \neq 0, \infty$ exists. The three sets of points satisfying ii), iii) and iv) were introduced in [Do92] and [CaPo97]. The Twist Theorem shows that these sets have measure 0 . If $\lim _{r \rightarrow 1} f^{\prime}(r \zeta)$ fails to exist on a set of positive measure then Plessner's Theorem for Bloch functions [Po92, p. 140] shows that assumption (1.9) is automatically satisfied. The special case of Corollary 1.2 that $\lim f^{\prime}(r \zeta)$ exists almost nowhere is contained in [CaPo97, Theorem 1]. The boundary point $f(\zeta)$ is called well-accessible [Po92, p. 251] if there is a curve $z(t), 0 \leq t \leq 1$ with $z(0)=\zeta$ such that

$\operatorname{diam}\{f(z(\tau)): t \leq \tau \leq 1\}=O(\operatorname{dist}(f(z(t)), \partial f(\mathbb{D}))), \quad$ as $t \longrightarrow 1$

It is known $[\mathrm{CaPo} 97,(3.17)]$ that the condition

$$
-b \leq \log \left|f^{\prime}(r \zeta)\right| \leq b, \quad b>1
$$

implies that $f(\zeta)$ is well-accessible and [CaPo97, (3.18)] that

$$
\left|\arg f^{\prime}(r \zeta)-\arg (f(r \zeta)-f(\zeta))\right| \leq c_{3} b .
$$


Proof of Corollary 1.2. Let $n>c_{1}$; see (1.3). By (1.9) there exist $r_{n}<1$ such that $a_{n}=\log f^{\prime}\left(r_{n} \zeta_{0}\right)$ satisfies $\operatorname{Re} a_{n}<-16 n$. We define

$$
\varphi_{n}(z)=\frac{z+r_{n} \zeta_{0}}{1+r_{n} \bar{\zeta}_{0} z}, f_{n}=f \circ \varphi_{n}, g_{n}=\frac{1}{8}\left(\log f^{\prime} \circ \varphi_{n}-a_{n}\right)
$$

Then $g_{n} \in \mathcal{B}$ with $g_{n}(0)=0$ and $\left\|g_{n}\right\|_{\mathcal{B}} \leq 1$ by (1.6). We apply Theorem 1.1 with $G=\left\{\operatorname{Re} w<\left|\operatorname{Re} a_{n}\right|\right\}, R=n$ and curves

$$
\Gamma_{j}(t), \quad 0 \leq t<1(j=1,2,3,4)
$$

such that $\Gamma_{j}(0)=0, \operatorname{Re} \Gamma_{j}(t)=0$ and, as $t \longrightarrow 1$,

i) $\lim \inf \operatorname{Im} \Gamma_{1}(t)=-\infty, \lim \sup \operatorname{Im} \Gamma_{1}(t)=+\infty$,

ii) $\left.\lim \operatorname{Im} \Gamma_{2}(t)\right)=+\infty$,

iii) $-\infty<\liminf \operatorname{Im} \Gamma_{3}(t)<+\infty, \lim \sup \operatorname{Im} \Gamma_{3}(t)=+\infty$,

iv) $\lim \inf \operatorname{Im} \Gamma_{4}(t)=0, \lim \sup \operatorname{Im} \Gamma_{4}(t)=3 \pi+2 n+\left(c_{3} b_{n}+\left|a_{n}\right|\right) / 8$,

see (1.15) below. Then (1.3) is satisfied, and (1.2) holds by (1.8) because $\left|\operatorname{Re} a_{n}\right|>16 n$. We conclude that there are sets $E_{j n} \subset \mathbb{T}$ with

$$
\operatorname{dim} E_{j n} \geq 1-\frac{c_{2}}{n}, \quad \text { for } j=1, \ldots, 4 \text { and } n>c_{1},
$$

such that (1.5) holds for $\zeta \in E_{j n}$. We obtain from (1.12) that

$$
\log f_{n}^{\prime}(z)=a_{n}+\log \left(\left(1-r_{n}^{2}\right)\left(1+\bar{\zeta}_{0} r_{n} z\right)^{-2}\right)+8 g_{n}(z) .
$$

Since $\operatorname{Re} \gamma_{\zeta}(r)=0$ it follows from (1.5) that

$$
|\log | f_{n}^{\prime}(r \zeta)|| \leq b_{n}:=\left|\operatorname{Re} a_{n}\right|+\log \frac{1+r_{n}}{1-r_{n}}+16 n
$$

so that $f_{n}(\zeta)$ is well-accessible; see (1.10). We obtain from (1.5), (1.11) and (1.15) that

$$
\begin{aligned}
\limsup _{r \rightarrow 1} \mid \arg \left(f_{n}(\right. & \left.r \zeta)-f_{n}(\zeta)\right)-8 \gamma_{\zeta}(r) \mid \\
& <16 n+c_{3} b_{n}+\left|\operatorname{Im} a_{n}\right|+2 \\
& <\infty
\end{aligned}
$$


for $\zeta \in E_{j n}$. Finally we set

$$
E_{j}=\bigcup_{n} \varphi_{n}\left(E_{j n}\right), \quad j=1,2,3,4
$$

Then $\operatorname{dim} E_{j}=1$ by (1.13), and if $\zeta \in E_{j}$ then $\zeta=\varphi_{n}\left(\zeta_{n}\right)$ for some $\zeta_{n} \in E_{j n}$.

Hence $f(\zeta)=f_{n}\left(\zeta_{n}\right)$ is well-accessible, and by the Koebe distortion theorem it is easy to deduce from (1.16) and the choice of $\operatorname{Im} \Gamma_{j}(t)$ that $\alpha(\zeta)$ and $\beta(\zeta)$ have the required properties.

REMARK 1. We assume now that $f(\mathbb{D})$ is bounded by a rectifiable curve. Then $f^{\prime} \in H^{1}$ and thus [Du70, p. 24]

$$
f^{\prime}(z)=e^{i \alpha} \exp \left(\frac{1}{2 \pi} \int_{\mathbb{T}} \frac{\zeta+z}{\zeta-z} \log \left|f^{\prime}(\zeta)\right||d \zeta|\right) \exp \left(-\int_{\mathbb{T}} \frac{\zeta+z}{\zeta-z} d \mu(\zeta)\right),
$$

where $\mu \geq 0$ is a singular measure. By definition $f(\mathbb{D})$ is a Smirnov domain if $\mu=0$. Hence (1.8) holds if $\left|f^{\prime}(\zeta)\right| \geq 1$ for almost all $\zeta \in \mathbb{T}$, and (1.9) holds if $f(\mathbb{D})$ is not a Smirnov domain. In particular Corollary 1.2 can be applied if $f(\mathbb{D})$ is a Keldish-Lavrentiev domain, that is a non-Smirnov domain for which $\left|f^{\prime}(\zeta)\right|=1$ for almost all $\zeta \in \mathbb{T}$; see [DuShSh66].

REMARK 2. There are local versions of Theorem 1.1 and Corollary 1.2. We can replace $\mathbb{T}$ by an open subarc $A$ and restrict $\zeta$ and our sets $E$ to lie in $A$.

\section{The proof of Theorem 1.1.}

We use the martingale technique introduced by Makarov [Ma90] into the theory of Bloch functions. For $n=0,1, \ldots$ let $\mathcal{D}_{n}$ be the family of dyadic arcs of length $2 \pi / 2^{n}$ on $\mathbb{T}$, that is,

$$
\mathcal{D}_{n}=\left\{\left\{e^{i t}: \frac{2 \pi k}{2^{n}} \leq t<\frac{2 \pi(k+1)}{2^{n}}\right\}: 0 \leq k<2^{n}\right\} .
$$

If $I$ and $J$ are any dyadic arcs then $I \cap J=\varnothing$ or $I \subset J$ or $J \subset I$. Let $g \in \mathcal{B}$ and $n=0,1, \ldots$ We define the martingale associated to $g$ by

$$
W_{n}(\zeta) \equiv W_{n}(I)=\lim _{r \rightarrow 1} \frac{1}{|I|} \int_{I} g(r s)|d s|, \quad \text { for } \zeta \in I \in \mathcal{D}_{n},
$$


where $|\cdot|$ denotes the linear measure on $\mathbb{T}$. Let $c_{1}, c_{2}, \ldots$ denote suitable positive absolute constants. We need two known results. The first is due to Makarov [Ma90]; compare [Po92, p. 156].

Proposition 2.1 (Makarov). Let $g \in \mathcal{B},\|g\|_{\mathcal{B}} \leq 1$ and let $W_{n}$ be the associated martingale. Then

$$
\begin{gathered}
\left|g(r \zeta)-W_{n}(\zeta)\right|<c, \quad \text { for } \zeta \in \mathbb{T}, 1-\frac{1}{2^{n}} \leq r \leq 1-\frac{1}{2^{n+1}} \\
\left|W_{n+1}(\zeta)-W_{n}(\zeta)\right|<c, \quad \text { for } \zeta \in \mathbb{T} .
\end{gathered}
$$

We also need the following technical result [ON95], [Do97]; compare [Ro93, p. 493].

Proposition 2.2 (O'Neill, Donaire). Let $W_{n}$ be the martingale associated to $g \in \mathcal{B}$ and let $\|g\|_{\mathcal{B}} \leq 1,0<\alpha<\pi / 2$. Let $I \in \mathcal{D}_{m}$ and $R>c_{1}(\alpha)$. If the stopping time

$$
\tau_{I}(\zeta)=\inf \left\{n>m:\left|W_{n}(\zeta)-W_{m}(\zeta)\right| \geq R\right\}
$$

is finite for almost all $\zeta \in I$, then

$$
\left|\left\{\zeta \in I:\left|\arg \left(W_{\tau_{I}(\zeta)}(\zeta)-W_{m}(\zeta)\right)-\vartheta\right|<\alpha\right\}\right| \geq c_{2}(\alpha)|I|
$$

for every $\vartheta$. Here $c_{1}(\alpha)$ and $c_{2}(\alpha)$ only depend on $\alpha$.

Proof of Theorem 1.1. a) Let $\Gamma(t), 0 \leq t<1$ be some parametrization of our given curve $\Gamma$. Let $\mathcal{F}_{0}=\{\mathbb{T}\}$ and $t_{0}=0$. We shall recursively construct families $\mathcal{F}_{j}$ of dyadic arcs such that each arc in $\mathcal{F}_{j}$ is contained in some arc of $\mathcal{F}_{j-1}$, furthermore stopping times

$$
t_{j}(\zeta) \equiv t_{j}(I) \in[0,1], \quad \text { for } \zeta \in I \in \mathcal{F}_{j-1}
$$

constant on $I$ such that $t_{j-1}(\zeta) \leq t_{j}(\zeta)$ and

$$
\operatorname{dist}\left(W_{m}(I), \mathbb{C} \backslash G\right)>R+c, \quad \text { for } I \in \mathcal{F}_{j} \cap \mathcal{D}_{m}
$$

where $c$ is the constant of Proposition 2.1. 
b) Suppose that $\mathcal{F}_{j}$ and $t_{j}$ have already been defined. Let $\zeta \in I \in$ $\mathcal{F}_{j}$. Then $I \in \mathcal{D}_{m}$ for some $m$. If $t_{j}(\zeta)=1$ then we define $t_{j+1}(\zeta)=1$, otherwise

$$
t_{j+1}(\zeta) \equiv t_{j+1}(I)=\inf \left\{t>t_{j}(\zeta):\left|\Gamma(t)-W_{m}(I)\right| \geq R\right\},
$$

if this set is empty we define $t_{j+1}(I)=1$ and $A_{j}(I)=I$.

Now let $t_{j+1}(I)<1$. Plessner's theorem for Bloch functions [Po92, p. 140] says that, for almost all $\zeta \in \mathbb{T}$, either the radial limit $g(\zeta)$ exists or the limit set of $g(r \zeta)$ as $r \longrightarrow 1$ is equal to $\hat{\mathbb{C}}$. Hence it follows from assumption (1.2) that

$$
\liminf _{r \rightarrow 1} \operatorname{dist}(g(r \zeta), \mathbb{C} \backslash G)=0, \quad \text { for almost all } \zeta \in \mathbb{T},
$$

so that, by (2.3),

$$
\liminf _{n \rightarrow \infty} \operatorname{dist}\left(W_{n}(\zeta), \mathbb{C} \backslash G\right) \leq c, \quad \text { for almost all } \zeta \in \mathbb{T} .
$$

Therefore we obtain from (2.4) and (2.8) that, for almost all $\zeta \in I$, the stopping time $\tau_{I}(\zeta)$ defined in $(2.5)$ is finite. By (2.4) we then have

$$
R \leq\left|W_{\tau_{I}(\zeta)}(\zeta)-W_{m}(\zeta)\right|<R+c .
$$

Thus we can apply Proposition 2.2 with $\alpha=1 / 4$. We see from (2.6) that, for $R>c_{3}=\max \left\{4 c, c_{1}\right\}$, the set

$$
\begin{aligned}
A_{j}(I)=\{\zeta \in I: & \mid \arg \left(W_{\tau_{I}(\zeta)}(\zeta)-W_{m}(\zeta)\right) \\
& \left.-\arg \left(\Gamma_{t_{j+1}(I)}-W_{m}(I)\right) \mid<\frac{1}{4}\right\}
\end{aligned}
$$

satisfies $\left|A_{j}(I)\right| \geq c_{2}|I|$. Note that $A_{j}(I)$ is the union of dyadic arcs $J \in \mathcal{D}_{n}$ with $n>m$.

We define $\mathcal{F}_{j+1}$ as the family of the dyadic arcs $J$ of $A_{j}(I)$ for all $I \in \mathcal{F}_{j}$. Then

$$
\sum_{\substack{J \subset I \\ J \in \mathcal{F}_{j+1}}}|J|=\left|A_{j}(I)\right| \geq c_{2}|I| .
$$

Furthermore it follows from (2.4) and (2.10) that $\tau_{I}(\zeta) \geq m+R / c$. Hence

$$
J \in \mathcal{F}_{j+1}, J \subset I \in \mathcal{F}_{j} \text { implies }|J| \leq 2^{-R / c}|I|
$$


Now we verify (2.8) for $j+1$, that is, we shall show that

$$
\operatorname{dist}\left(W_{n}(J), \mathbb{C} \backslash G\right)>R+c,
$$

for $J \in \mathcal{F}_{j+1}, \zeta \in I \in \mathcal{F}_{j}, n=\tau_{I}(\zeta)$; see (2.11). This is trivial by (2.8) if $t_{j+1}(I)=1$ and thus $A_{j}(I)=I$. Therefore let $t_{j+1}(I)<1$. Since $\Gamma(t)$ is continuous we see from $(2.9)$ that $\left|\Gamma\left(t_{j+1}(I)\right)-W_{m}(I)\right|=R$. Hence it follows from (2.10) and (2.11) that the quantity

$$
q=\frac{W_{n}(\zeta)-W_{m}(\zeta)}{\Gamma_{t_{j+1}}(I)-W_{m}(I)}
$$

satisfies $1 \leq|q| \leq 1+c / R$ and $|\arg q|<1 / 4$. Since $R>c_{3} \geq 4 c$ we deduce that $|q-1|<1 / 2$. Hence

$$
\left|W_{n}(\zeta)-\Gamma\left(t_{j+1}\right)\right|=\left|\Gamma\left(t_{j+1}\right)-W_{m}(\zeta)\right||q-1|<\frac{R}{2}
$$

and it follows by assumption (1.3) that

$$
\operatorname{dist}\left(W_{n}(\zeta), \mathbb{C} \backslash G\right) \geq \operatorname{dist}(\Gamma, \partial G)-\frac{R}{2} \geq \frac{3 R}{2}>R+c .
$$

This completes our construction.

c) We define

$$
E_{\Gamma}=\bigcap_{j \geq 1} \bigcup_{I \in \mathcal{F}_{j}} I
$$

It follows from (2.12) and (2.13) by a theorem [Po92, p. 226] of Hungerford [Hu88] and Makarov [Ma90] that

$$
\operatorname{dim} E_{\Gamma} \geq \frac{\log \left(c_{2} 2^{R / c}\right)}{\log 2^{R / c}}=1-\frac{c \log \left(\frac{1}{c_{2}}\right)}{R \log 2}
$$

which proves (1.4).

Now let $\zeta \in E_{\Gamma}$. There are two cases.

i) First we assume that $t_{j}(\zeta)<1$ for all $j$. Let $I_{j} \in \mathcal{F}_{j}$ be the arc containing $\zeta$. Then $I_{j} \in \mathcal{D}_{n_{j}}$ for some $n_{j}$. We define $\varphi_{\zeta}:[0,1) \longrightarrow[0,1)$ by $\varphi_{\zeta}\left(2^{-n_{j}}\right)=t_{j}(\zeta)$ and linear in between. We parametrize $\Gamma$ by 
$\gamma_{\zeta}(r)=\Gamma\left(\varphi_{\zeta}(r)\right), 0 \leq r<1$. If $1-2^{-n_{j}} \leq r \leq 1-2^{-n_{j+1}}$ then $t_{j}(\zeta) \leq \varphi_{\zeta}(r) \leq t_{j+1}(\zeta)$ and thus

$\left|g(r \zeta)-\gamma_{\zeta}(r)\right| \leq\left|g(r \zeta)-W_{n_{j}}(\zeta)\right|+\left|\Gamma\left(\varphi_{\zeta}(r)\right)-W_{n_{j}}\left(I_{j}\right)\right| \leq c+R \leq 2 R$

by (2.3) and (2.9).

ii) Now we suppose that $t_{j}(\zeta)<1$ for $j \leq k$ and $t_{j}(\zeta)=1$ for $j>k$. Then we define $\varphi_{\zeta}$ as in (i) for $j<k$ but linear in $\left[1-2^{-n_{k}}, 1\right]$. If $1-2^{-n_{k}} \leq r<1$ then (see (2.9))

$$
\left|\Gamma\left(\varphi_{\zeta}(r)\right)-W_{n}(\zeta)\right|<R, \quad \text { for } n \geq n_{k}
$$

and (1.5) follows as above.

\section{Balanced Bloch functions.}

Let $\triangle(\zeta, \rho)$ denote the non-euclidean disk of center $\zeta \in \mathbb{D}$ and radius $\rho$. For $g \in \mathcal{B}$ we define

$$
\mu_{g}(r)=\sup _{r \leq|z|<1}\left(1-|z|^{2}\right)\left|g^{\prime}(z)\right|, \quad 0 \leq r<1 .
$$

Using the maximum principle for $|z| \leq r$, we see that

$$
\left|g^{\prime}(z)\right| \leq \max \left\{\frac{\mu_{g}(r)}{1-r^{2}}, \frac{\mu_{g}(r)}{1-|z|^{2}}\right\}, \quad \text { for } z \in \mathbb{D}, 0 \leq r<1 \text {. }
$$

By definition we have $g \in \mathcal{B}_{0}$ if $\mu_{g}(r) \longrightarrow 0$ as $r \longrightarrow 1$.

We call $g$ a balanced Bloch function if there exist $a>0$ and $\rho<\infty$ such that

$$
\sup _{z \in \triangle(\zeta, \rho)}\left(1-|z|^{2}\right)\left|g^{\prime}(z)\right| \geq a \mu_{g}(|\zeta|), \quad \text { for } \zeta \in \mathbb{D} .
$$

This condition is trivially satisfied if $0<\alpha \leq\left|g^{\prime}(z)\right| \leq \beta<\infty$ for $z \in \mathbb{D}$. Balanced Bloch functions for the case $g \notin \mathcal{B}_{0}$ were first considered by P. Jones [Jo89]; see e.g. also [Ro91], [BiJo97]. Jones showed that if $J=\partial f(\mathbb{D})$ is a quasicircle, then $\log f^{\prime}$ is balanced and not in $\mathcal{B}_{0}$ if and only if

$$
\inf _{w_{1}, w_{2} \in J} \sup \left\{\frac{\left|w_{1}-w\right|+\left|w-w_{2}\right|}{\left|w_{1}-w_{2}\right|}: w \in J \text { between } w_{1} \text { and } w_{2}\right\}>1 .
$$


Curves with this property are called uniformly wiggly. The prototype of balanced Bloch functions are sufficiently regular series with Hadamard gaps.

Theorem 3.1. Suppose that

$$
\begin{gathered}
1<\lambda \leq \frac{n_{k+1}}{n_{k}} \leq \lambda^{\prime}<\infty, \quad \text { for } k=0,1, \ldots \\
\frac{1}{M}\left(\frac{n_{j}}{n_{k}}\right)^{\alpha}\left|b_{j}\right| \leq\left|b_{k}\right| \leq M\left|b_{j}\right|, \quad \text { for } 0 \leq j \leq k
\end{gathered}
$$

with constants $M$ and $\alpha<1$. Then

$$
g(z)=\sum_{k=0}^{\infty} b_{k} z^{n_{k}}, \quad z \in \mathbb{D},
$$

is a balanced Bloch function.

A typical example of a balanced Bloch function is

$$
g(z)=\sum_{k=1}^{\infty} k^{-\gamma} z^{2^{k}}, \quad 0 \leq \gamma<\infty .
$$

Proof. Let $M_{1}, M_{2}, \ldots$ denote constants that depend only on $\lambda, \lambda^{\prime}, \alpha$ and $M$. If $1-1 / n_{j} \leq r \leq 1-1 / n_{j+1}$ and $|z|=r$ then, by (3.6),

$$
\begin{aligned}
\left|z g^{\prime}(z)\right| & \leq \sum_{k=0}^{j} n_{k}\left|b_{k}\right|+\sum_{k=j+1}^{\infty} n_{k}\left|b_{k}\right| \exp \left(-\frac{n_{k}}{n_{j+1}}\right) \\
& \leq M n_{j}^{\alpha}\left|b_{j}\right| \sum_{k=0}^{j} n_{k}^{1-\alpha}+\lambda^{\prime} M n_{j}\left|b_{j}\right| \sum_{k=j+1}^{\infty} \frac{n_{k}}{n_{j+1}} \exp \left(-\frac{n_{k}}{n_{j+1}}\right)
\end{aligned}
$$

by (3.5) and (3.4). Since $t e^{-t}$ is decreasing for $t \geq 1$ we therefore obtain from (3.4) that

$$
\left|z g^{\prime}(z)\right| \leq M_{1} n_{j}\left|b_{j}\right|+\lambda^{\prime} M n_{j}\left|b_{j}\right| \sum_{\nu=0}^{\infty} \lambda^{\nu} \exp \left(-\lambda^{\nu}\right) \leq M_{2} \frac{\left|b_{j}\right|}{\left(1-r^{2}\right)} .
$$

Using the maximum principle near $z=0$, we thus see from (3.1) that

$$
\mu_{g}(r) \leq \sup _{k \geq j} M_{3}\left|b_{k}\right| \leq M_{4}\left|b_{j}\right|, \quad \text { for } 1-\frac{1}{n_{j}} \leq r \leq 1-\frac{1}{n_{j+1}}
$$


Now we apply a standard method [Bi69] to estimate the coefficients of gap series. It follows from (3.4), (3.5) and [GHPo87, Theorem 2] that

$$
n_{j}\left|b_{j}\right| \leq M_{5} \sup \left\{\left|g^{\prime}(z)\right|: z \in \triangle(\zeta, \rho)\right\} \text {, }
$$

for $1-M_{6} / n_{j} \leq|\zeta| \leq 1-M_{7} / n_{j}$. Hence

$$
\sup _{z \in \triangle(\zeta, \rho)}\left(1-|z|^{2}\right)\left|g^{\prime}(z)\right| \geq M_{8}^{-1}\left(1-|\zeta|^{2}\right) n_{j}\left|b_{j}\right| \geq M_{9}^{-1} \mu_{g}(r)
$$

by (3.7).

Further examples of balanced Bloch functions come from automorphic forms. Let $\Gamma$ be a Fuchsian group with compact fundamental domain $F$ in $\mathbb{D}$. Let $h$ be an analytic automorphic form of weight 1 , corresponding to a differential on the Riemann surface $\mathbb{D} / \Gamma$. Then $\gamma^{\prime} h \circ \gamma=h$ for $\gamma \in \Gamma$ and

$$
g(z)=\int_{0}^{z} h(\zeta) d \zeta, \quad z \in \mathbb{D}
$$

is a balanced Bloch function because $\bar{F} \subset \mathbb{D}$. Note that inf $\mu_{g}(r)>0$.

Now we prove two results on real convex functions needed for the next section.

Lemma 3.2. Let the real-valued functions $\varphi$ and $\psi$ be continuous and convex in the interval $I \subset \mathbb{R}$. If the function

$$
\chi(s)=\sup _{t \geq s}(\varphi(t)-\psi(t))+\psi(s), \quad s \in I
$$

is finite, then it is also continuous and convex in $I$.

ProOF. The function $\sup \{\varphi(t)-\psi(t): t \in I, t \geq s\}$ is decreasing in $s \in I$. Let $I_{k}=\left[s_{k}, t_{k}\right]$ be its intervals of constancy with values $c_{k}$. We define

$$
\chi_{k}(s)= \begin{cases}\varphi(s), & \text { for } s \in I \backslash I_{k} \\ c_{k}+\psi(s), & \text { for } s \in I_{k}\end{cases}
$$

Since $\varphi(s)-\psi(s) \leq c_{k}$ for $s \in I_{k}$, we have

$$
\varphi(s) \leq c_{k}+\psi(s)=\chi_{k}(s), \quad \text { for } s_{k} \leq s \leq t_{k},
$$


with equality for $s=s_{k}$ and $s=t_{k}$. The convex function $\varphi$ has left and right derivatives $D^{ \pm} \varphi$ in $I$ and $D^{ \pm} \varphi$ is increasing [HLP67, p. 91-94]. If $s<s_{k}$ then

$$
D^{+} \chi_{k}(s)=D^{+} \varphi(s) \leq D^{+} \varphi\left(s_{k}\right) \leq D^{+} \psi\left(s_{k}\right)=D^{+} \chi_{k}\left(s_{k}\right)
$$

by (3.10). If $s_{k} \leq s<t_{k}$ then

$$
D^{+} \chi_{k}\left(s_{k}\right)=D^{+} \psi\left(s_{k}\right) \leq D^{+} \psi(s)=D^{+} \chi_{k}(s)
$$

by (3.9). Since $D^{-} \psi\left(t_{k}\right) \leq D^{-} \varphi\left(t_{k}\right)$ by (3.10), we furthermore have

$$
D^{+} \chi_{k}(s) \leq D^{-} \psi\left(t_{k}\right) \leq D^{-} \varphi\left(t_{k}\right) \leq D^{+} \varphi\left(t_{k}\right)=D^{+} \chi_{k}\left(t_{k}\right) .
$$

Using again that $D^{+} \varphi$ and $D^{+} \psi$ are increasing, we deduce that $D^{+} \chi_{k}$ is increasing in $I$. Since $\chi_{k}$ is locally absolutely continuous it follows by integration that $\chi_{k}$ is convex. Finally $\chi=\sup _{k} \chi_{k}$ by (3.9) and (3.10), so $\chi$ is also convex.

Lemma 3.3. The function

$$
\chi(s)=\log \mu_{g}\left(e^{s}\right)-\log \left(1-e^{2 s}\right), \quad-\infty<s<0
$$

is convex and the function $u(z)=\chi(\log |z|)$ with $u(0)=\log \mu_{g}(0)$ is continuous and subharmonic in $\mathbb{D}$.

Proof. Let $M(r)=\max \left\{\left|g^{\prime}(z)\right|:|z|=r\right\}$. It follows from (3.1) that (3.8) holds with

$$
\varphi(s)=\log M\left(e^{s}\right), \quad \psi(s)=-\log \left(1-e^{2 s}\right) .
$$

The function $\varphi$ is convex by the Hadamard three circles theorem [Co78, p. 137], and $\chi$ is convex because $\psi^{\prime \prime}(s)=4 e^{2 s}\left(1-e^{2 s}\right)^{-2}>0$. Therefore $\chi$ is convex by Lemma 3.2. It follows that $u$ is subharmonic [HaKe76, Theorem 2.2].

\section{Properties of balanced Bloch functions.}

Let $\mu_{g}$ be defined by (3.1). We consider the open level sets

$$
A_{g}(\varepsilon)=\left\{z \in \mathbb{D}:\left(1-|z|^{2}\right)\left|g^{\prime}(z)\right|<\varepsilon \mu_{g}(|z|)\right\},
$$


for $0<\varepsilon \leq 1$. We see from (4.1) and (3.1) that

$$
\left|g^{\prime}(z)\right| \geq \frac{\varepsilon \mu_{g}(r)}{1-r^{2}} \geq \varepsilon \max _{|\zeta|=r}\left|g^{\prime}(\zeta)\right|, \quad \text { for } z \notin A_{g}(\varepsilon),|z|=r .
$$

If $g^{\prime}$ is unbounded it follows that $\mathbb{T} \subset \overline{A_{g}(\varepsilon)}$ for all $\varepsilon>0$. Otherwise we would have $\left|g^{\prime}(z)\right| \longrightarrow \infty$ as $z \longrightarrow I$ for some arc $I$ of $\mathbb{T}$, which is impossible by the Privalov uniqueness theorem [Po92, p. 140].

Let $M_{1}, \ldots$ denote positive constants that depend only on $a$ and $\rho$ in the definition (3.3) of balanced Bloch functions. In particular, if $g^{\prime}$ is unbounded then $A_{g}(\varepsilon)$ is nonempty for $0<\varepsilon \leq 1$. By contrast, the example $g(z) \equiv z$ shows that $A_{g}(\varepsilon)$ can be empty if $g^{\prime}$ is bounded and $\varepsilon<1$.

Proposition 4.1. Let $g$ be a balanced Bloch function and let $z_{0} \in \mathbb{D}$. Then the harmonic measure satisfies

$$
\omega\left(z_{1}, \bar{\triangle}\left(z_{0}, 2 \rho\right) \cap \bar{A}_{g}(\varepsilon), \triangle\left(z_{0}, 2 \rho\right) \backslash \bar{A}_{g}(\varepsilon)\right) \leq \frac{M_{1}}{\log \left(\frac{1}{\varepsilon}\right)},
$$

for some $z_{1} \in \triangle\left(z_{0}, \rho\right)$.

Proof. We write $r=\left|z_{0}\right|, \triangle_{0}=\triangle\left(z_{0}, 2 \rho\right)$ and $A=\bar{A}_{g}(\varepsilon)$. It follows from (3.2) that

$$
\left|g^{\prime}(z)\right| \leq \frac{M_{2}}{1-r^{2}} \mu_{g}(r), \quad \text { for } z \in \bar{\triangle}_{0} .
$$

It follows from (4.1) that

$$
\left|g^{\prime}(z)\right| \leq \frac{M_{2}}{1-r^{2}} \mu_{g}(r) \varepsilon, \quad \text { for } z \in \bar{\triangle}_{0} \cap A .
$$

Hence the two-constants theorem [Ah73, p. 39] implies that

$$
\left|g^{\prime}(z)\right| \leq \frac{M_{2}}{1-r^{2}} \mu_{g}(r) \varepsilon^{\omega\left(z, \bar{\triangle}_{0} \cap A, \triangle_{0} \backslash A\right)},
$$

for $z \in \triangle_{0} \backslash A$. By (3.3) there exists $z_{1} \in \triangle\left(z_{0}, \rho\right)$ such that

$$
\left|g^{\prime}\left(z_{1}\right)\right| \geq \frac{a}{1-\left|z_{1}\right|^{2}} \mu_{g}(r) \geq \frac{M_{3}^{-1}}{1-r^{2}} \mu_{g}(r) .
$$


Hence (4.2) follows from (4.4).

Theorem 4.2. Let $g$ be a balanced Bloch function. Then there are $\alpha>0$ and $\varepsilon_{0}>0$ such that every component of $A_{g}(\varepsilon)\left(0<\varepsilon<\varepsilon_{0}\right)$ lies in some disk $\triangle\left(z_{0}, \varepsilon^{\alpha}\right)\left(z_{0} \in \mathbb{D}\right)$ and contains a zero of $g^{\prime}$.

Proof. a) Let $B$ be a component of $A_{g}(\varepsilon)$, let $z_{0} \in B$ and let $B_{0}$ be the component of $B \cap \triangle\left(z_{0}, \rho / 2\right)$ with $z_{0} \in B_{0}$. Let $\varphi$ map $\triangle\left(z_{0}, 2 \rho\right) \backslash \bar{B}_{0}$ conformally onto $\{r<|z|<1\}$ such that $\partial \triangle\left(z_{0}, 2 \rho\right)$ corresponds to $\mathbb{T}$. Then

$$
\omega\left(z, \bar{\triangle}\left(z_{0}, 2 \rho\right) \cap \bar{B}_{0}, \triangle\left(z_{0}, 2 \rho\right) \backslash \bar{B}_{0}\right)=\frac{\log \left(\frac{1}{|\varphi(z)|}\right)}{\log \left(\frac{1}{r}\right)} .
$$

Since $B_{0} \subset A_{g}(\varepsilon)$ it follows from Proposition 4.1 and the principle of majorization for harmonic measure [Ah73, p.39] that

$$
\frac{\log \left(\frac{1}{\left|\varphi\left(z_{1}\right)\right|}\right)}{\log \left(\frac{1}{r}\right)} \leq \frac{M_{1}}{\log \left(\frac{1}{\varepsilon}\right)}
$$

for some $z_{1} \in \triangle\left(z_{0}, \rho\right)$. Since $B_{0} \subset \triangle\left(z_{0}, \rho / 2\right)$ a normal family argument gives $\left|\varphi\left(z_{1}\right)\right|<1-\alpha_{1}$ where $\alpha_{1}>0$ depends only on $a$ and $\rho$. Hence $r \leq \varepsilon^{\alpha_{2}}$ and therefore

$$
B_{0} \subset \triangle\left(z_{0}, \varepsilon^{\alpha}\right), \quad \text { for } 0<\varepsilon<\alpha_{3} \text {. }
$$

Since $B$ is connected and contains $z_{0}$, it follows that $B=B_{0}$ if $\varepsilon^{\alpha}<\rho / 2$.

b) Now we prove that every component $B$ of $A_{g}(\varepsilon)$ with $\bar{B} \subset \mathbb{D}$ contains a zero of $g^{\prime}$. Suppose that $g^{\prime}(z) \neq 0$ for $z \in B$ and thus for $z \in \bar{B}$. Then $\log \left|g^{\prime}\right|$ is harmonic in $B$ and continuous in $\bar{B}$. Hence it follows from Lemma 3.3 that

$$
v(z)=\log \mu_{g}(|z|)-\log \left(1-|z|^{2}\right)-\log \left|g^{\prime}(z)\right|
$$

is subharmonic in $B$ and continuous in $\bar{B}$. Since $B$ is a component of $A_{g}(\varepsilon)$ and since $\bar{B} \subset \mathbb{D}$, we see from (4.1) that $v(z)=\log (1 / \varepsilon)$ for $z \in \partial B$ and thus $v(z) \leq \log (1 / \varepsilon)$ for $z \in B$ by the maximum principle for subharmonic functions. But this contradicts (4.1). 
Theorem 4.3. Let $g$ be a balanced Bloch function and suppose that

$$
\frac{\mu_{g}\left(r^{\prime}\right)}{\mu_{g}(r)} \geq \frac{1-r^{\prime}}{1-r} \lambda\left(\frac{1-r}{1-r^{\prime}}\right), \quad \text { for } 0<r<r^{\prime}<1
$$

where $\lambda(x) \nearrow \infty$ as $x \longrightarrow \infty$. Then there exist $\varepsilon>0$ and $\rho^{*}<\infty$ such that every disk $\triangle\left(\zeta, \rho^{*}\right)(\zeta \in \mathbb{D})$ contains a component of $A_{g}(\varepsilon)$.

Some (rather weak) condition like (4.5) is necessary as the balanced Bloch function $g(z) \equiv z$ shows. Note that (4.5) implies that $g^{\prime}$ is unbounded.

Proof. We claim: Given $\varepsilon>0$ there exists $\rho^{\prime}<\infty$ such that

$$
\triangle\left(\zeta, \rho^{\prime}\right) \cap A_{g}(\varepsilon) \neq \varnothing, \quad \text { for every } \zeta \in \mathbb{D}
$$

This claim implies the assertion of Theorem 4.3 with $\rho^{*}=\rho^{\prime}+2 \varepsilon^{\alpha}$ and $0<\varepsilon<\varepsilon_{0}$ by Theorem 4.2.

Suppose our claim is false. Then, for $0<\varepsilon<1$, there exist $z_{n} \in \mathbb{D}$ such that

$$
\left(1-|z|^{2}\right)\left|g^{\prime}(z)\right|>\varepsilon \mu_{g}(|z|), \quad \text { for } z \in \triangle\left(z_{n}, n\right), n=1,2, \ldots
$$

We write $r_{n}=\left|z_{n}\right|$ and consider the functions

$$
h_{n}(s)=\frac{1-r_{n}^{2}}{\mu_{g}\left(r_{n}\right)} g^{\prime}\left(\frac{s+z_{n}}{1+\bar{z}_{n} s}\right), \quad s \in \mathbb{D} .
$$

It follows from (4.8) and (3.2) that $\left|h_{n}(s)\right| \leq 4 /\left(1-|s|^{2}\right)$ for $s \in \mathbb{D}$. Therefore we may assume that $h_{n} \longrightarrow h$ as $n \longrightarrow \infty$ locally uniformly in $\mathbb{D}$. Furthermore we may assume that $z_{n} \longrightarrow \zeta \in \mathbb{T}$.

Let $|s|=\sigma<1$. By (3.1) and (4.5) we have

$$
\mu_{g}\left(\left|\frac{s+z_{n}}{1+\bar{z}_{n} s}\right|\right) \geq \mu_{g}\left(\frac{\sigma+r_{n}}{1+r_{n} \sigma}\right) \geq \frac{1-\sigma}{1+r_{n} \sigma} \lambda\left(\frac{1+r_{n} \sigma}{1-\sigma}\right) \mu_{g}\left(r_{n}\right)
$$

Hence it follows from (4.7) and (4.8) that

$$
\left|h_{n}(s)\right| \geq \frac{\varepsilon\left|1+\bar{z}_{n} s\right|^{2}}{(1+\sigma)\left(1+r_{n} \sigma\right)} \lambda\left(\frac{1+r_{n} \sigma}{1-\sigma}\right) .
$$


Since $h_{n} \longrightarrow h$ and $\zeta_{n} \longrightarrow \zeta$ as $n \longrightarrow \infty$, we conclude that

$$
|h(s)| \geq \frac{\varepsilon|1+\bar{\zeta} s|^{2}}{(1+\sigma)^{2}} \lambda\left(\frac{1+\sigma}{1-\sigma}\right) \geq \frac{\varepsilon}{4} \lambda\left(\frac{1+\sigma}{1-\sigma}\right),
$$

for $\operatorname{Re}(\bar{\zeta} s)>0$. Hence

$$
|h(s)| \longrightarrow \infty, \quad \text { as }|s| \longrightarrow 1,
$$

$\operatorname{Re}(\bar{\zeta} s)>0$ which contradicts the Privalov uniqueness theorem $[\operatorname{Pr} 56$, p. 208], [Po92, p. 140].

Geometric interpretation. Let $g$ be a balanced Bloch function that satisfies condition (4.5). Let $\varepsilon>0$ be small but fixed. Then

$$
\left|g^{\prime}(z)\right| \geq \varepsilon \frac{\mu_{g}(|z|)}{1-|z|^{2}} \longrightarrow \infty, \quad \text { as }|z| \longrightarrow 1, z \in \mathbb{D} \backslash A_{g}(\varepsilon)
$$

by (4.5). Theorem 4.2 says that the components of $A_{g}(\varepsilon)$ have small hyperbolic diameter, each containing a zero of $g^{\prime}$, whereas Theorem 4.3 says that there are many components. Hence the surface

$$
\left\{(x, y, u): x+i y \in \mathbb{D}, u=\left|g^{\prime}(x+i y)\right|\right\}
$$

rises to infinity at $\partial \mathbb{D}$ except for very many very small but deep holes near the zeros of $g^{\prime}$.

Ruscheweyh and Wirths [RuWi82] have studied, for any Bloch function $g$, the set where $\left(1-|z|^{2}\right)\left|g^{\prime}(z)\right|$ attains its maximum and its relation to the zeros of $g^{\prime}$.

J. Becker [Be87], [PoWa82, Theorem 4.2] has shown that, for any $g \in \mathcal{B}$, the condition

$$
\int_{0}^{1} \mu_{g}(r)^{2} \frac{d r}{1-r}<\infty
$$

implies that $g \in$ VMOA (vanishing mean oscillation) and thus has finite radial limits $g(\zeta)$ for almost all $\zeta \in \mathbb{T}$. It follows [Pr56, p. 208] that $\operatorname{cap}\{g(\zeta): \zeta \in \mathbb{T}, g(\zeta) \neq \infty$ exists $\}>0$.

Now we turn to a condition stronger than (4.10), namely

$$
\int_{0}^{1} \mu_{g}(r) \frac{d r}{1-r}<\infty .
$$


It follows from (3.1) by integration that $\int_{0}^{1}\left|g^{\prime}(r \zeta)\right| d r<\infty$ for all $\zeta \in \mathbb{T}$ and that $g$ is continuous in $\overline{\mathbb{D}}$. We show now that exactly the opposite happens if $g \in \mathcal{B}$ is balanced and condition (4.11) is false.

Theorem 4.4. Let $g$ be a balanced Bloch function with

$$
\int_{0}^{1} \mu_{g}(r) \frac{d r}{1-r}=\infty
$$

If $C$ is any curve in $\mathbb{D}$ ending on $\mathbb{T}$, then

$$
\int_{C}\left|g^{\prime}(z)\right||d z|=\infty
$$

Furthermore $g$ assumes every value in $\mathbb{C}$ infinitely often in $\mathbb{D}$.

Geometric interpretation. Let $g$ be a balanced Bloch function that satisfies (4.10) and (4.12). The Riemann image surface of $g$ over $\mathbb{C}$ then has many accessible boundary points; their projection to $\mathbb{C}$ has positive capacity. But (4.13) shows that none of these boundary points is accessible through a curve of finite length.

Proof. Let $c_{1}, c_{2}, \ldots$ denote suitable positive constants. Since $C$ goes to $\mathbb{T}$, we can find $z_{n} \in C, r_{n} \nearrow 1$ and disks $\triangle_{n}$ such that

$$
\triangle_{n}=\triangle\left(z_{n}, 2 \rho\right) \subset\left\{r_{n}<|z|<r_{n+1}\right\}, \quad \frac{1-r_{n+1}}{1-r_{n}}>c_{1} .
$$

Let $\varphi_{n}$ map $\triangle_{n}$ conformally onto $\mathbb{D}$ such that $\varphi_{n}\left(z_{n}\right)=0$. By Proposition 4.1 there exist $\varepsilon>0$ and $z_{n}^{*} \in \triangle\left(z_{n}, \rho\right)$ such that

$$
\frac{M_{1}}{\log \left(\frac{1}{\varepsilon}\right)}>\omega\left(z_{n}^{*}, \bar{\triangle}_{n} \cap \bar{A}_{g}(\varepsilon), \triangle_{n} \backslash \bar{A}_{g}(\varepsilon)\right)=\omega\left(s_{n}^{*}, A_{n}, \mathbb{D} \backslash A_{n}\right),
$$

where $s_{n}^{*}=\varphi_{n}\left(z_{n}^{*}\right)$ and $A_{n}=\varphi_{n}\left(\bar{\triangle}_{n} \cap \bar{A}_{g}(\varepsilon)\right)$. If $p_{n}$ denotes the circular projection onto the radius from 0 to $\mathbb{T}$ opposite to $s_{n}^{*}$, then [Ah73, p. 43], [Ne53, p. 108]

$$
\omega\left(s_{n}^{*}, p_{n}\left(A_{n}\right), \mathbb{D} \backslash p_{n}\left(A_{n}\right)\right)<\frac{M_{1}}{\log \left(\frac{1}{\varepsilon}\right)} .
$$


Since $s_{n}^{*} \in \varphi_{n}\left(\triangle\left(z_{n}, \rho\right)\right)=\left\{|z|<\rho^{*}\right\}$ with $\rho^{*}<1$ depending only on $\rho$, we see that the linear measure satisfies $\left|p_{n}\left(A_{n}\right)\right|<M_{4} / \log (1 / \varepsilon)$. Since $\varphi_{n}\left(C \cap \triangle_{n}\right)$ connects 0 and $\mathbb{T}$, we conclude that

$$
\left|\varphi_{n}\left(C \cap \triangle_{n}\right) \backslash A_{n}\right| \geq 1-\left|p_{n}\left(A_{n}\right)\right|>1-\frac{M_{4}}{\log \left(\frac{1}{\varepsilon}\right)}>\frac{1}{2}
$$

if $\varepsilon$ is chosen sufficiently small. It is easy to deduce that

$$
\left|\left(C \cap \triangle_{n}\right) \backslash A_{g}(\varepsilon)\right|>c_{1}\left(1-\left|z_{n}\right|\right)>c_{1} c_{2}\left(1-r_{n}\right)
$$

by (4.14). Hence it follows from (4.1) that

$$
\int_{C \cap \triangle_{n}}\left|g^{\prime}(z)\right||d z| \geq \frac{\varepsilon \mu_{g}\left(r_{n+1}\right)}{1-r_{n}^{2}}\left|\left(C \cap \triangle_{n}\right) \backslash A_{g}(\varepsilon)\right|>\frac{\varepsilon c_{2}}{2} \mu_{g}\left(r_{n+1}\right) .
$$

Since $\mu_{g}(r)$ is decreasing we have

$$
\sum_{n} \mu_{g}\left(r_{n}\right) \geq c_{1} \sum_{n} \int_{r_{n}}^{r_{n+1}} \frac{\mu_{g}(r)}{1-r} d r=\infty
$$

by (4.14) and (4.12). This implies (4.13).

The last assertion is an immediate consequence of (4.13) and the following proposition, where $g$ need not be a Bloch function.

Proposition 4.5. Let $g$ be analytic in $\mathbb{D}$ and suppose that (4.13) holds for any curve $C$ in $\mathbb{D}$ ending on $\mathbb{T}$. Then $g$ assumes every finite value infinitely often in $\mathbb{D}$.

Proof. a) For $w \in \mathbb{C}$ let $N(w) \leq \infty$ denote the number of zeros (with multiplicity) of $g-w$ in $\mathbb{D}$. Let $w, w^{\prime} \in \mathbb{C}$ and let $L$ be a rectifiable Jordan arc from $w$ to $w^{\prime}$ that does not meet $\left\{g(z): z \in \mathbb{D}, g^{\prime}(z)=0\right\}$ except possibly in $w$ and $w^{\prime}$. At each point $z_{k}$ of $g^{-1}(\{w\})$, we consider the maximal Jordan $\operatorname{arcs} C_{k}$ in $g^{-1}(L)$ with initial point $z_{k}$; the number of these arcs is equal to the multiplicity of the zero $z_{k}$ of $g-w$. Therefore there are $N(w)$ arcs $C_{k}$ altogether.

The maximal arc $C_{k}$ ends either at some point $z_{k}^{\prime} \in \mathbb{D}$ with $g\left(z_{k}^{\prime}\right)=$ $w^{\prime}$ or approaches $\mathbb{T}$. The second case cannot arise by our assumption because $\left|g\left(C_{k}\right)\right| \leq|L|<\infty$. The number of points $z_{k}^{\prime}$ that coincide is 
equal to the multiplicity of $g-w^{\prime}$ in $z_{k}^{\prime}$. Hence $N\left(w^{\prime}\right) \geq N(w)$ and thus $N\left(w^{\prime}\right)=N(w)$ by symmetry. Thus we have shown

$$
N(w) \equiv m \leq \infty, \quad \text { for } w \in \mathbb{C} .
$$

b) Now we give a proof of the known fact that, for any function $g$ analytic in $\mathbb{D}$, it is not possible that (4.15) holds with $m<\infty$. Let

$$
r(\rho)=\sup \{|z|:|g(z)|=\rho\}, \quad 0<\rho<\infty .
$$

We claim that $r(\rho)<1$. Otherwise there would exist $w$ with $|w|=\rho$ and points $z_{n} \in \mathbb{D}$ with $\left|z_{n}\right| \longrightarrow 1$ such that $g\left(z_{n}\right) \longrightarrow w$. But $w$ is assumed $m$ times in $\mathbb{D}$ so that there exist distinct $z_{n_{k}}(k=1, \ldots, m)$ with $g\left(z_{n_{k}}\right)=g\left(z_{n}\right)$ and $z_{n_{k}} \neq z_{n}$ for large $n$, which would imply $N(w)>m$.

It follows from (4.16) that $|g(z)| \neq \rho$ in $R(\rho)=\{r(\rho)<|z|<1\}$. Since $g(R(\rho))$ is an unbounded domain we conclude that $|g(z)|>\rho$ for $z \in R(\rho)$ for any $\rho>0$. Hence $|g(z)| \longrightarrow \infty$ as $|z| \longrightarrow 1$, which contradicts the Privalov uniqueness theorem.

Acknowledgement. We are very grateful to the referee for having pointed out that three mathematical arguments in the original version of this section were wrong.

\section{References.}

[Ah73] Ahlfors, L. V., Conformal invariants. McGraw Hill, 1973.

[Be72] Becker, J., Löwnersche Differentialgleichung und quasikonform fortsetzbare schlichte Funktionen. J. Reine Angew. Math. 255 (1972), 23-43.

[Be87] Becker, J., On asymptotically conform extension of univalent functions. Complex Variables 9 (1987), 109-120.

[Bi69] Binmore, K. G., Analytic functions with Hadamard gaps. Bull. London Math. Soc. 1 (1969), 211-217.

[BiJo97] Bishop, C. J., Jones, P. W., Wiggly sets and limit sets. Arkiv. Math. 35 (1997), 201-224.

[CaPo97] Carmona, J. J., Pommerenke, Ch., Twisting behaviour of conformal maps. J. London Math. Soc. 56 (1997), 16-36.

[Co78] Conway, J. B., Functions of a complex variable. Springer, 1978. 
[Do92] Donaire, J. J., Comportamiento en la frontera de la derivada de una representación conforme. Master Thesis. Univ. Aut. Barcelona, 1992.

[Do97] Donaire, J. J., On the radial behaviour of inner functions in $\mathcal{B}_{0}$. Preprint. Univ. Aut. Barcelona, 1997.

[Du70] Duren, P. L., Theory of $H^{p}$ spaces. Academic Press, 1970.

[DuShSh66] Duren, P. L., Shapiro, H. S., Shields, A. L., Singular measures and domains not of Smirnov type. Duke Math. J. 33 (1966), 247-254.

[Fa85] Falconer, K. J., The geometry of fractal sets. Cambridge Univ. Press, 1985.

[GHPo87] Gnuschke-Hauschild, D., Pommerenke, Ch., On dominance in Hadamard gap series. Complex Variables 9 (1987), 189-197.

[HaKe76] Hayman, W. K., Kennedy, P. B., Subharmonic functions. Vol. I. Academic Press, 1976.

[HLP67] Hardy, G. H., Littlewood, J. E., Pólya, G., Inequalities. Cambridge Univ. Press, 1967.

[Hu88] Hungerford, G. J., Boundaries of smooth sets and singular sets of Blaschke products in the little Bloch space. Thesis. CalTech, 1988.

[Jo89] Jones, P. W., Square functions, Cauchy integrals, analytic capacity and Hausdorff measure. Lecture Notes in Math. Springer 1384 (1989), 2468.

[Ma90] Makarov, N. G., Probability methods in the theory of conformal mapping. Leningrad Math. J. 1 (1990), 1-56.

[Mc69] McMillan, J. E., Boundary behavior of a conformal mapping. Acta Math. 123 (1969), 43-67.

[Ne53] Nevanlinna, R., Eindeutige analytische Funktionen. Second Ed. Springer, 1953.

[ON95] O'Neill, M. D., Some results on $H^{\infty}$ and the Bloch space. Thesis. Univ. of California, 1995.

[Po92] Pommerenke, Ch., Boundary behaviour of conformal maps. Springer, 1992.

[PoWa82] Pommerenke, Ch., Warschawski, S. E., On the quantitative boundary behavior of conformal maps. Comm. Math. Helvetici 57 (1982), 107129.

[Pr56] Privalov, I. I., Randeigenschaften analytischer Funktionen. Deutscher Verlag Wiss., 1956.

[Ro91] Rohde, S., On conformal welding and quasicircles. Michigan Math. J. 38 (1991), 111-116.

[Ro93] Rohde, S., The boundary behaviour of Bloch functions. J. London Math. Soc. 48 (1993), 488-499. 
[RuWi82] Ruscheweyh, S., Wirths, K.-J., On extreme Bloch functions with prescribed critical prints. Math. Z. 180 (1982), 91-105.

Recibido: 26 de junio de 1.997

Revisado: 20 de mayo de 1.998

Juan Jesús Donaire* Departament de Matemàtiques Universitat Autònoma de Barcelona E-08193 Bellaterra, SPAIN donaire@manwe.mat.uab.es

and

Christian Pommerenke Fachbereich Mathematik Technische Universität Berlin D-10623 Berlin, GERMANY pommeren@math.tu-berlin.de

* Partially supported by a CIRIT grant (Generalitat de Catalunya) and by DGICYT grant PB-95-0956-C02-02. Part of this research was done at Technische Universität Berlin. 